\title{
The diversity of first foods offered at weaning is predicted by indices of maternal diet quality, breastfeeding and parity
}

\author{
J.E. Miller ${ }^{1}$, L.F. Foglizzo ${ }^{1}$, C.C. Crook ${ }^{1}$ and J.M. Wallace ${ }^{1}$ \\ ${ }^{1}$ Rowett Institute, University of Aberdeen, Aberdeen, UK
}

Lifelong eating behaviours and food preferences are shaped by experiences throughout infancy and childhood ${ }^{(1)}$. Thus, it is important to establish healthy habits early on. Mothers are usually the main gatekeepers of the range of first foods offered to their babies ${ }^{(2)}$. Their approach to weaning may be influenced by their own dietary habits, choices around breast versus formula-feeding and prior experience: accordingly, the diversity and sequence of foods offered during the three months after first solid food introduction has been analysed in relation to these parameters.

Maternal diet was assessed by questionnaire which yielded fruit and vegetable (F\&V) frequency and diversity scores, and average portions per day consumed. Milk-feeding approach for six-months postnatal was based on monthly questionnaires. Mothers $(\mathrm{n}=59)$ recorded each new food offered per month, and whether the food was liked/disliked. A hybrid classification system was developed based on the strengths of UK, USA and Australian nutritional guidelines allowing for detailed analysis of foods offered across six main and 52 sub-food groups. Fifty-eight percent of mothers were primiparous, $69 \%$ predominately breastfed and $36 \%$ had a prepregnancy $\mathrm{BMI}>25 \mathrm{~kg} / \mathrm{m} 2$.

Babies began weaning at $24.6 \pm 1.78$ weeks (mean $\pm \mathrm{sd}$ ) and were offered a median of 48 different foods (range23-82). These comprised vegetables $(32.4 \%)$, fruits $(21.5 \%)$, protein foods $(17.9 \%)$, grain/grain products $(15.4 \%)$, dairy/dairy alternatives $(6.3 \%)$ and noncore foods $(6.5 \%)$. Sixty-six percent of mothers offered two or more different non-core foods. Eighty-nine percent of total novel foods offered were liked, independent of main food group $(\mathrm{P}>0.2$, chi squared). Month influenced the number of new fruits, vegetables, grain and dairy foods offered $(\mathrm{P}=0.002$ to $<0.001)$, with more in month one than month three (mixed effect repeated measures). Stepwise multiple regression revealed that mothers' portions F\&V per day, primiparity and breastfeeding predicted the total number of different $\mathrm{F} \& \mathrm{~V}$ offered to infants $(\beta 2.35 \mathrm{P}=0.021, \beta 4.38 \mathrm{P}=0.016, \beta 4.40 \mathrm{P}=0.029$, respectively). Conversely, the number of noncore foods offered (primarily confectionary, cakes and biscuits) increased as mothers' $\mathrm{F} \& \mathrm{~V}$ diversity score decreased $(\beta-0.093, \mathrm{P}=$ $0.024)$. The latter score $(\max =50)$ predicted the number of nuts/seeds $(\beta 0.041, P=0.009)$, oily fish $(\beta 0.029, P=0.042)$ and 'other' grains $(\beta 0.051, \mathrm{P}=0.003)$, and whether sweetened $(\beta-0.018, \mathrm{P}=0.009)$, or non-sweetened yogurt $(\beta 0.025, \mathrm{P}=0.004)$ was offered. The diversity of $\mathrm{F} \& \mathrm{~V}$ consumed by the mother also predicted the infants' overall liking of foods offered $(\beta 0.283, \mathrm{P}=0.025)$. Finally, mothers with $\mathrm{BMI}>25 \mathrm{~kg} / \mathrm{m} 2$ offered fewer dark green vegetables $(\mathrm{P}=0.039)$, citrus fruits $(\mathrm{P}=0.033)$ and beans $/$ peas $(\mathrm{P}=0.040)$.

Thus, first-time mothers and those who consume a wide range of $\mathrm{F} \& \mathrm{~V}$, are a healthy weight, and choose to breastfeed, are more likely to offer their infants a wide range of healthful foods and fewer non-core foods. These maternal influences on the diversity of the diet offered to the infant at weaning have implications for establishing healthy food preferences throughout the life-course.

\section{Acknowledgements}

Funded by the Scottish Government's Rural and Environment Science and Analytical Services Division

\section{References}

1. Birch L \& Doub A (2014) Am J Clin Nutr 99, 723s-728s.

2. Harrison M, Brodribb W \& Hepworth J (2017) Matern Child Nutr 13, e12360. 